\title{
Cellular profiles of induced sputum in children with stable cystic fibrosis: comparison with BAL
}

\author{
N. Reinhardt*, C.I.U. Chen*, D. Loppow*, T. Schink ${ }^{*}$, I. Kleinau*, R.A. Jörres\# ${ }^{\#}$ U. Wahn*, \\ H. Magnussen", K.P. Paul*
}

Cellular profiles of induced sputum in children with stable cystic fibrosis: comparison with BAL. N. Reinhardt, C.I.U. Chen, D. Loppow, T. Schink, I. Kleinau, R. A.Jörres, U. Wahn, H. Magnussen, K.P. Paul. (C) ERS Journals Ltd 2003.

ABSTRACT: Neutrophil-dominated endobronchial inflammation is a major characteristic of cystic fibrosis (CF) and there is increasing demand for easy-to-perform noninvasive monitoring for prediction and intervention.

Fourteen stable paediatric CF patients (8-17 yrs; mean forced expiratory volume in one second $86.7 \%$ of the predicted value) were investigated once by fractional bronchoalveolar lavage (BAL) and by sputum induction on three occasions, 2-6 weeks apart. Sputum was induced by consecutive 10-min inhalations of 3, 4 and 5\% saline. CF sputum cellular profiles were compared with BAL fluid cell counts and samples from agematched healthy children, and between different time points to assess reproducibility.

Adequate sputum was recovered on $>\mathbf{9 5} \%$ of occasions. In all sputum fractions, CF patients showed higher neutrophil counts than healthy children. Neutrophil percentages were highest in the first BAL fraction (median 92\%), followed by sputum, in which the percentages decreased in consecutive fractions $(72,66$ and $64 \%)$, whereas counts were lowest in the pooled BAL fraction (53\%). Increasing percentages of macrophages mirrored the decreases in neutrophil percentage. Results of sputum induction at different time points in the $\mathrm{CF}$ patients showed good reproducibility and nonoverlap with counts from healthy children.

In conclusion, the results of sputum induction in children with mild stable cystic fibrosis adequately describe airway inflammation by providing cellular profiles with lower relative neutrophil counts than in the first ("bronchial") bronchoalveolar lavage fraction and higher relative neutrophil counts than in subsequent pooled ("more peripheral") bronchoalveolar lavage fractions.

Eur Respir J 2003; 22: 497-502.
Depts of *Paediatric Pneumology and Immunology, Charité, ${ }^{\top}$ Medical Biometry, Humboldt University, Berlin and ${ }^{\#}$ Centre for Pneumology and Thoracic Surgery, Großhansdorf Hospital, Großhansdorf, Germany.

Correspondence: K.P. Paul, Tanenwai 32, 25946 Nebel, Amrum, Germany.

Fax: 4930450566983

E-mail: karl.paul@lva-scheswig-holstein.de

Keywords: Bronchoalveolar lavage cell differentials

cystic fibrosis

induced sputum

macrophages

neutrophils

Received: May 242002

Accepted after revision: April 142003

This study was supported by grants from Mukoviszidose eV (Bonn, Germany) and Hoffmann-La Roche Company (GrenzachWyhlen, Germany).
In cystic fibrosis $(\mathrm{CF})$, endobronchial inflammation starts early in life and is closely related to destruction of the lungs [1]. Its most prominent feature is persistent neutrophilic infiltration. Early anti-inflammatory intervention might prevent these processes that result in an irreversible loss of lung function. Therefore, early detection of endobronchial inflammation is mandatory in CF. Bronchoalveolar lavage (BAL), although widely used in infants and children with $\mathrm{CF}$ for research purposes, is an invasive procedure and, therefore, less well-suited for monitoring in the paediatric setting than in adults $[2,3]$. BAL is also designed to preferentially sample the peripheral airspaces of the lungs, minimising contamination from the bronchi, although the first aliquot recovered from BAL contains a higher proportion of bronchial components than subsequent aliquots [4]. Since the mid-1990's, however, sputum induction has gained importance as a sensitive and reliable noninvasive tool in a number of airway diseases other than $\mathrm{CF}$, yielding results comparable with those of BAL [4-8].

Previous studies of induced sputum in children with CF did not include comparison with the first and subsequent pooled BAL aliquots $[9,10]$. As part of a prospective, multicentric study, the present authors' group has performed BAL examinations in CF patients with mild disease (forced expiratory volume in one second (FEV1) $>80 \%$ of the predicted value), aged $>5$ yrs [3]. In virtually all children, neutrophilic airway inflammation was shown in the first BAL aliquot $[3,11]$, indicating its diagnostic value in $\mathrm{CF}$.

In order to assess the usefulness of sputum induction, children from the Hospital of the Charite, Berlin, Germany, aged $<18$ yrs were selected and differential cell counts from consecutive saline inhalations analysed, since these might sample regions of increasing depth within the bronchial tree $[12,13]$. More specifically, the aim of the present study was to compare the inflammatory cell profiles of consecutive sputum samples with profiles from BAL fluid and sputum from agematched healthy children, as well as to assess the reproducibility of sputum cell counts over three repeated sputum inductions.

\section{Patients and methods}

\section{Patients}

Fourteen children with CF were included. All had taken part in the Bronchoalveolar Lavage for Evaluation of Anti-inflammatory Treatment (BEAT) study, a multicentric trial designed to monitor the progression of endobronchial inflammation by repeated BAL examinations that had started 3 yrs before the present study. Inclusion criteria had been 
FEV1 $>80 \%$ pred, no inhaled or systemic steroids, no antiinflammatory drugs (e.g. ibuprofen), and no serious organ involvement.

Sputum examinations were accepted by 14 out of 18 patients who had recently undergone the third of three BAL examinations within the BEAT study. Four patients declined, mostly due to a lack of time. The clinical data of the participating patients at the time of the first sputum test are summarised in table 1. Mean \pm SD FEV1 36 months previously had been $88.5 \pm 15.7 \%$ pred and 18 months previously $87.9 \pm 20.2 \%$ pred. Before BAL, patients were asked about their clinical status via a standardised questionnaire including questions on temperature changes, sputum colour, weight loss and cough. The answers to all these questions regarding deterioration in the patients' clinical status were negative 6 weeks before the tests. Only four patients had been able to expectorate spontaneously more than once at 3-monthly check-ups during the year before the first sputum induction. Furthermore, none had received courses of regular i.v. antibiotic therapy. On-demand i.v. treatment had to have been finished 6 weeks before the last BAL, which served as a reference for the sputum tests. Five of the patients had been treated with recombinant human deoxyribonuclease for $\geqslant 3$ yrs.

In order to reduce the likelihood of changes between BAL and sputum induction, as well as to avoid artefacts from the preceding BAL or sputum induction, the interval between BAL and sputum induction as well as between repeated sputum induction was 2-6 weeks [13]. In addition, patients were required to show no fever, weight loss, increase in sputum volume or change in sputum colour, dyspnoea, hypoxia or loss of appetite before each sputum induction. None of the patients had to be excluded due to such causes. Furthermore, no patient

Table 1.-Characteristics of cystic fibrosis patients and healthy children

\begin{tabular}{lcc}
\hline & Patients & Controls \\
\hline Males:females n & $6: 8$ & $12: 13$ \\
Age yrs & $13.1 \pm 3.1$ & $12.1 \pm 2.5$ \\
FEV1 \% pred & $86.7 \pm 17.9$ & $105.7 \pm 14.6$ \\
MEF25\% pred & $49.5 \pm 25.5$ & $110.4 \pm 34.8$ \\
Induced sputum & & \\
$\quad$ Cell viability \% & $80.3(74.5-91.7)$ & $84.7(81.5-90.5)$ \\
Total cell count & & \\
$10^{6}$ cells $\cdot \mathrm{mL}^{-1}$ & $3.5(2.1-8.0)$ & $1.9(0.7-3.2)$ \\
$3 \%$ saline & $3.0(1.9-4.6)$ & $1.3(0.8-2.6)$ \\
$4 \%$ saline & $5.0(3.1-6.1)$ & $1.1(0.6-2.7)$ \\
$5 \%$ saline & $245.9(174.3-412.9)$ & $14.8(8.0-40.7)$ \\
Neutrophils & $67.0(60.5-73.8)$ & $8.8(6.3-13.7)$ \\
$10^{4}$ cells $\cdot \mathrm{mL}^{-1}$ & $113.4(66.6-179.6)$ & $126.5(81.2-235.0)$ \\
$\%$ & $26.0(17.7-33.6)$ & $85.1(81.2-89.1)$ \\
Macrophages & $22.9(12.7-33.9)$ & $6.0(4.2-13.3)$ \\
$10^{4}$ cells $\cdot \mathrm{mL}^{-1}$ & $5.1(3.8-8.5)$ & $4.6(3.3-5.0)$ \\
$\%$ & $5.85(3.05-8.82)$ & $0.17(0.00-0.80)$ \\
Lymphocytes & & \\
$10^{4}$ cells $\cdot \mathrm{mL}^{-1}$ & $1.61(0.85-2.11)$ & $0.07(0.00-0.41)$ \\
$\%$ &
\end{tabular}

Data are presented as mean \pm SD or median (interquartile range). Sputum composition data are for the first induction after bronchoalveolar lavage in children with cystic fibrosis and expressed as pooled values (mean over consecutive inhalations of 3, 4 and $5 \%$ saline). FEV1: forced expiratory volume in one second; MEF25: maximum expiratory flow when $25 \%$ of the forced vital capacity remains to be exhaled; $\%$ pred: percentage of the predicted value. received courses of i.v. or changes in oral antibiotic therapy during the study period.

The control subjects comprised 25 age-matched children who were characterised by a physical examination and questionnaire to exclude any respiratory illness or upper respiratory tract infection. Bronchoscopy was not performed in these children.

The study was approved by the Ethics Committee of Charité and informed consent was obtained from all children and/or their parents according to legal standards.

\section{Bronchoalveolar lavage}

All BEAT study patients who participated in the sputum induction procedure had undergone bronchoscopy and fractional BAL with a $3.5-\mathrm{mm}(<12 \mathrm{yrs})$ or $4.9-\mathrm{mm}$ ( $\geqslant 12 \mathrm{yrs})$ flexible bronchoscope according to a standardised protocol carried out by the same investigator (K.P. Paul) [3, 12]. In short, $20-\mathrm{mL}$ fractions of $0.9 \%$ saline were instilled and immediately recovered up to a volume of $3 \mathrm{~mL} \cdot \mathrm{kg}$ body weight ${ }^{-1}$. BAL fluid cytology was examined separately for the first fraction and subsequent fractions (termed "pooled sample") as described previously [3]. BAL fluid epithelial cells (ciliated and squamous) accounted for $<5 \%$ of cells in the first sample and $<2 \%$ in the pooled fraction and were excluded from the cell count.

\section{Sputum induction and processing}

Sputum examinations in $\mathrm{CF}$ patients were performed on average 2.3 weeks after BAL and twice thereafter at intervals of $\sim 3.2$ weeks. The group of healthy children was tested once by sputum induction. All children were examined at the same time of day.

In order to facilitate comparison with literature data, a validated protocol that included prior inhalation of $200 \mu \mathrm{g}$ salbutamol and repeated spirometry to minimise the risk of bronchoconstriction was adhered to $[13,14]$. The hypertonic saline was inhaled in increasing concentrations (3, 4 and 5\%) from an ultrasonic nebuliser (Schill Company, Probstzella, Germany) for two 5-min periods at each concentration.

Each 5-min period was followed by expectoration of sputum and spirometry. Lung function was also assessed before sputum induction. Sputum samples were pooled for each saline concentration. Pooling over concentrations was carried out subsequently by computing the mean results.

Adequate sputum samples were separated macroscopically from saliva by visual assessment using a microscope (the more solid and opaque portions are sputum and are selected from the remainder, which is mainly saliva) and processed within $2 \mathrm{~h}$. (They were considered adequate when epithelial (squamous and ciliated) cell numbers were $<5 \%$ of the total and the total volume was $>1.5 \mathrm{~mL}$. Volumes of $<1.5 \mathrm{~mL}$ and samples with $>5 \%$ epithelial cells were accepted on the basis of microscopic quality). After weighing, samples were suspended 1:2 in sputolysin $\mathbb{R}$ (weight/weight), incubated for $15 \mathrm{~min}$ at $37^{\circ} \mathrm{C}$ and washed in phosphate-buffered saline (PBS; $\mathrm{pH} 7.4$ ) by centrifugation for $10 \mathrm{~min}$ at $600 \times g$. The cells were then resuspended in PBS containing 1\% bovine serum albumin. Cell counts were assessed using a haemocytometer and viability by trypan blue exclusion. Cytocentrifuge slides (MiniZentrifuge RF; Heraeus, Hanau, Germany) were prepared from $20,000-40,000$ cells and 400-500 cells were counted by one experienced investigator (N. Reinhardt) for differential cell counts (excluding epithelial cells). Sputum bacteriology was 
not included since there had been agreement between throat swabs and BAL fluid bacteriology in all but two patients.

\section{Statistical analysis}

Nonparametric repeated-measures analyses of variance were used to take into account the correlated nature of the repeated measurements [15]. For comparison of healthy controls and patients, two-factorial analysis was performed for each cell type, with group and saline concentration as factors. One-factorial analysis was used to compare the fractional patterns of absolute and relative cell counts obtained by BAL as well as sputum induction. Reproducibility was examined by two-factorial analysis, with time and saline concentration as factors. Further, intraclass correlation coefficients were determined to quantify reproducibility [16]. A p-value of 0.05 was considered significant.

\section{Results}

\section{Safety and efficacy of bronchoalveolar lavage and sputum induction}

BAL did not lead to complications and was well tolerated. Similarly, sputum induction proved to be safe in the children studied, as described previously for adults [16]. The maximum fall in FEV1 during induction was $\sim 4.8 \%$ and never exceeded $20 \%$; none of the tests had to be stopped because of bronchoconstriction. All of the CF patients produced sufficient sputum during all three consecutive 10 -min inhalation periods but four of 25 healthy children only did so at one or two saline concentrations. The weight of sputum samples in $\mathrm{CF}$, as a mean over the three visits, was $1.1 \mathrm{~g}$ after $3 \%$ saline, $1.2 \mathrm{~g}$ after $4 \%$ and $1.4 \mathrm{~g}$ after $5 \%$. Pseudomonas aeruginosa was recovered from the BAL fluid of seven patients, Staphylococcus aureus from five and both organisms from two.

\section{Bronchoalveolar lavage fluid cytology}

Cell viability (by trypan blue exclusion) was $79 \%$ in the first fraction and $80 \%$ in the pooled sample of BAL fluid. The corresponding total cell counts were $48 \times 10^{4}$ and $60 \times 10^{4}$ cells $\cdot \mathrm{mL}^{-1}$. In accordance with results obtained across the whole BEAT population [3], the patients participating in the present study demonstrated a greater percentage of neutrophils in the first fraction compared to the pooled sample: neutrophils accounted for (median (range)) $92(0.4-96) \%$ of cells in the first BAL fraction and $53(2-93) \%$ in the pooled sample $(\mathrm{p}<0.01)$. The corresponding percentages of macrophages were 4 $(0.7-88) \%$ and $40(2-93) \%$, lymphocytes $3(1-11) \%$ and 6 $(1-18) \%$, and eosinophils $<1(0.2-2) \%$ in both fractions. Prior examination of the same 14 patients 36 and 18 months previously had shown relative median neutrophil counts of 61 and $77 \%$ in the first fraction of BAL fluid, respectively.

\section{Sputum cytology}

The viability of samples pooled across the three saline concentrations was similar in the two groups (CF patients and controls) studied (table 1).

Squamous epithelial cell contamination was $<20 \%$ in all cases and $<5 \%$ in $95 \%$ of cases. The mean epithelial cell count was $3.2 \%$ in $\mathrm{CF}$ patients and $3.9 \%$ in controls. Absolute numbers of neutrophils were significantly elevated in $\mathrm{CF}$ patients compared to healthy children $(\mathrm{p}<0.001)$. Median total counts in CF patients were $3.0 \times 10^{6}, 2.2 \times 10^{6}$ and $3.1 \times 10^{6}$ cells $\cdot \mathrm{mL}^{-1}$ after 3,4 and $5 \%$ saline, respectively. In contrast, counts were as low as $0.2 \times 10^{6}, 0.1 \times 10^{6}$ and $0.07 \times 10^{6}$ cells $\cdot \mathrm{mL}^{-1}$ in the control subjects (table 1 ).

Eosinophil counts were low in both groups but CF patients showed significantly higher counts than controls $(\mathrm{p}=0.001)$ (table 1). They also exhibited significantly higher lymphocyte counts $(\mathrm{p}<0.001)$ (table 1$)$. There was no significant difference between patients and controls regarding the absolute number of macrophages.

The percentages of neutrophils and macrophages in sputum induced by different saline concentrations in CF patients and controls are shown in figure 1. Neutrophil percentages were significantly elevated in CF patients (median 72, 66 and $64 \%$ ) compared to controls (median 11, 9 and $5 \% ; \mathrm{p}<0.001$ ) and there was almost no overlap between the two groups (fig. 1a).

Similarly, the percentage of macrophages differed between CF patients (median 16, 23 and 30\%) and controls (median 83,87 and $91 \% ; \mathrm{p}<0.001$ ) (fig. 1b). There were also significant differences between percentages of eosinophils $(\mathrm{p}<0.005)$ and lymphocytes $(\mathrm{p}<0.05)$ between $\mathrm{CF}$ patients and controls (table 1).
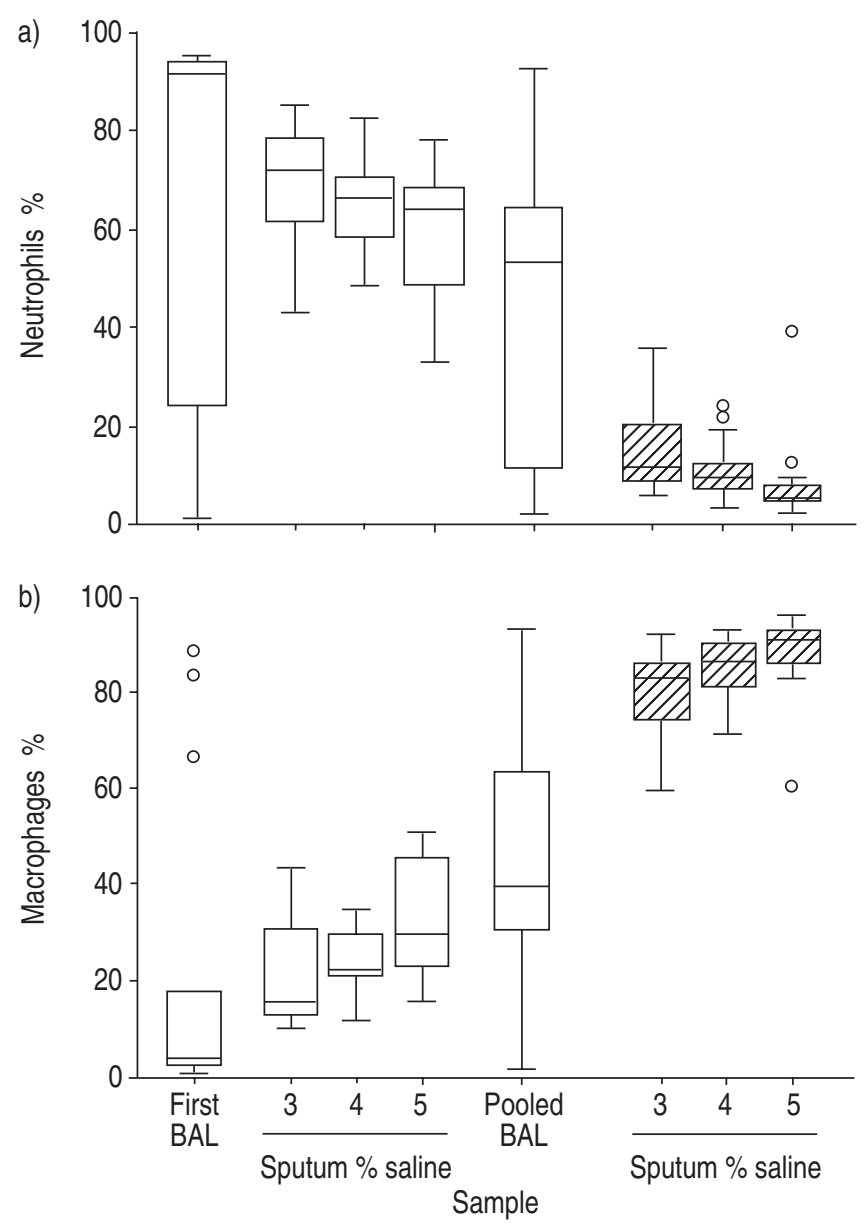

Fig. 1.-Boxplots showing percentages of a) neutrophils and b) macrophages in induced sputum from healthy children $(\mathbb{Z})$ and cystic fibrosis (CF) patients ( $\square$ ). The box represents the median and interquartile range; the whiskers show the minimum and maximum values excluding outliers (O). Data were derived from the first induction after bronchoalveolar lavage (BAL) and are given separately for consecutive inhalations of 3,4 and $5 \%$ saline. For comparison, values from the first fraction and pooled BAL fluid of $\mathrm{CF}$ patients at the lavage prior to the first sputum induction are also shown. 
The consecutive inhalation of 3,4 and $5 \%$ saline led to a consistent change in cellular profiles (fig. 1). In both healthy children $(\mathrm{p}<0.001)$ and $\mathrm{CF}$ patients $(\mathrm{p}<0.005)$, macrophages, as markers of peripheral airways and alveolar space, appeared in significantly increased numbers over consecutive inhalations (fig. 1b). Correspondingly, percentages of neutrophils decreased significantly $(\mathrm{p}<0.001$ and $\mathrm{p}<0.05)$ (fig. 1a).

\section{Bronchoalveolar lavage versus sputum induction}

Overall, cell densities were significantly lower in BAL fluid than in induced sputum $(\mathrm{p}<0.001)$. When comparing the percentage of neutrophils in BAL fluid with that in sputum in $\mathrm{CF}$, there was a consistent decrease from the first fraction of BAL, across the three induced sputum fractions and to the pooled BAL sample $(p<0.001)$ (fig. 1a). As a mirror image, the percentages of macrophages increased $(p<0.001)$ (fig. 1b). It was observed that two of the patients with normal differential cell counts in the pooled BAL fraction showed increases in neutrophil percentages in induced sputum. In these patients, neutrophil numbers were $1.70 \pm 1.84 \%$ for the first and $5.50 \pm 4.38 \%$ for the pooled BAL fraction. In induced sputum, the same patients showed neutrophil percentages of $69.33 \pm 11.31 \%$ for $3 \%$ saline, $60.66 \pm 17.29 \%$ for $4 \%$ and $64.65 \pm 10.39 \%$ for $5 \%$. As regards other inflammatory cells, only the percentage of eosinophils showed a significant difference between BAL fluid and induced sputum, being higher in sputum $(\mathrm{p}<0.05)$.

\section{Reproducibility}

In order to assess the reproducibility of sputum cell counts over time, sputum induction was repeated on three separate occasions in the CF patients at intervals of 3.1-3.4 weeks. Figure 2 depicts the individual percentages of neutrophils (fig. 2a) and macrophages (fig. 2b) for all CF patients at the different time points. Intraclass correlation coefficients for pooled percentages of macrophages were 0.67 between the first and second sputum induction $(\mathrm{p}<0.05), 0.80$ between the second and third $(\mathrm{p}<0.005)$, and 0.71 between the first and third $(\mathrm{p}<0.05)$. The corresponding values for neutrophils were $0.68(\mathrm{p}<0.05), 0.80(\mathrm{p}<0.005)$ and $0.76(\mathrm{p}<0.01)$. On nonparametric analysis of variance, no significant change over time could be seen for macrophages or neutrophils.

\section{Discussion}

The present study indicates that, in children with CF and mild lung disease, sputum induction yields a profile of inflammatory cells that corresponds well to the profile obtained by BAL. Fractional analysis of sputum samples collected after each of three consecutive inhalations with increasing saline concentrations further demonstrated a gradient in neutrophil and macrophage numbers, suggesting that more and more peripheral airways are sampled by prolonged inhalation. Although a similar trend could be observed in both $\mathrm{CF}$ patients and age-matched healthy controls, cellular profiles, nevertheless, reproducibly distinguished between the two groups. Sputum induction was well tolerated and all patients were capable of producing sputum after hypertonic saline inhalation [17]. The present results suggest that sputum induction is a valid and promising tool for clinical and research purposes in children with mild CF. Furthermore, data are provided on reproducibility over time, which is necessary for future therapeutic interventions.
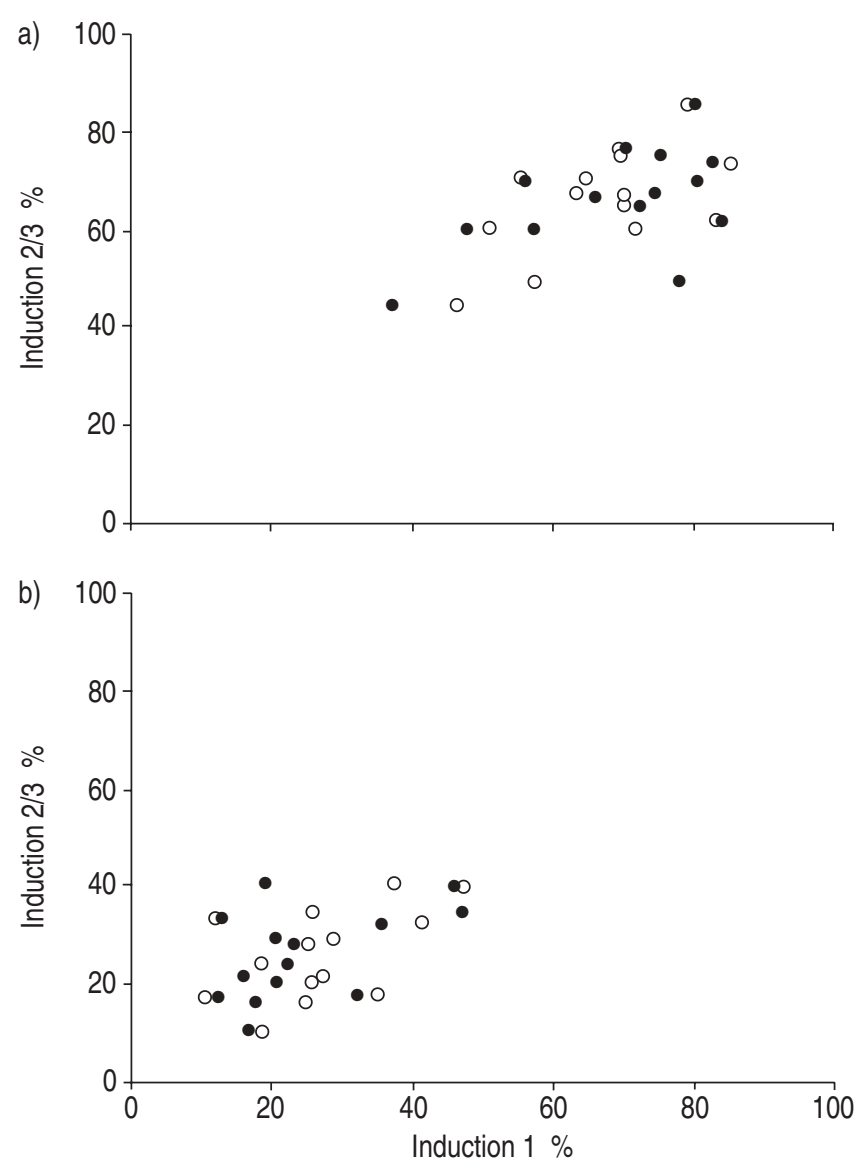

Fig. 2.-Individual percentages of a) neutrophils and b) macrophages in induced sputum. Data are presented as means over consecutive inhalations. In order to demonstrate reproducibility over time, values obtained in the second (O) and third ( $\bigcirc)$ inductions are plotted against those from first induction. On average, there were 3.1 weeks between the first and second, and 3.4 weeks between the second and third inductions.

To date, BAL has generally been considered the gold standard for the assessment of airway inflammation in children with CF. Accordingly, BAL fluid differential cell counts from the present patients demonstrated neutrophilic inflammation and were comparable to published data obtained using the same method $[3,11]$. The present evaluation of induced sputum represents the first comparison to BAL fluid in children with $\mathrm{CF}$. It showed that the percentage of neutrophils in induced sputum was within the range of the first and pooled fractions of BAL fluid.

Sputum differential cell counts from the age-matched healthy control group were similar to those described for adults in the literature [13]. Previous reports in this age group had already suggested that sputum samples obtained over consecutive saline inhalations supply information about airway inflammation at increasing depths in the bronchial tree [12, 13]. Indeed, the change in neutrophil numbers observed in the present study in children and adolescents indicates that the first BAL fraction and the first sputum sample originate from more central airways, and that the last sputum sample and pooled BAL sample are derived from more peripheral bronchi (fig. 1). The fact that there was virtually no overlap between $\mathrm{CF}$ patients and controls as regards the percentage of neutrophils in sputum is a strong argument that sputum induction samples a region of the bronchial tree that is highly informative about airway inflammation in $\mathrm{CF}$ and renders sputum induction potentially superior to BAL for this disease 
[18]. This is particularly relevant in view of the fact that fluid recovery in the first BAL fraction (often referred to as the "bronchial wash"), even in stable children with $\mathrm{CF}$, is quantitatively inferior to subsequent fractions and, in a considerable number of cases, does not yield sufficient material for analysis [3].

Despite this, the usefulness of sputum induction in $\mathrm{CF}$ could vary. When comparing BAL fluid with sputum from adult patients with more advanced disease after inhalation of $5 \%$ saline, reasonable agreement between inflammatory indices was found [19]. However, in this population, neutrophils accounted for $\geqslant 80 \%$ of all cells in both sputum and BAL samples, in accordance with the fact that endobronchial inflammation progresses with age. Such extreme values might pose a problem for the detection of therapeutic effects or monitoring disease progression. Therefore, surrogate inflammatory parameters that respond to changes in inflammation or damage of the airways and lungs would be useful. Conversely, the present data, not being that near to the upper limits, might be relevant for studies on the effect of interventions in patients with less advanced lung disease.

It is of note that only four of the CF children in the present population were capable of expectorating spontaneously more than once during 3-monthly check-ups in the year before the first sputum induction. All of them, however, produced sufficient material after saline inhalation. Hypertonic saline is indeed known to be a potent mobilising agent for secretions in this patient group and used therapeutically in cases otherwise refractory to therapy. However, regarding tolerability, it should be taken into account that all children were in a stable condition. Furthermore, the minimum age at which sputum induction can be performed was not the subject of the present study. All patients were aged $\geqslant 8$ yrs and applicability in younger children can only be speculated upon.

Sputum neutrophil and macrophage percentages were highly reproducible in children with mild $\mathrm{CF}$, as judged from the intraclass correlation coefficients. Since the three repeated sputum inductions were performed within 6-12 weeks, conclusions cannot be drawn regarding individual long-term monitoring of the disease. The data from BAL in patients with mild disease were obtained after exclusion of acute deterioration through strict clinical criteria [3]. The time interval between BAL and the first sputum induction was only 2.3 weeks and that between repeated sputum examinations 3.2 weeks, thereby rendering an effect of BAL on sputum induction or of sputum induction on subsequent inductions unlikely. As the confounding influence of different treatment modalities had been minimised and patients treated with antiinflammatory substances excluded, the present data on reproducibility are likely to provide an unbiased estimate of the short-term variability in sputum neutrophil percentages in the clinically stable children studied. Reproducibility, however, is essential not only for monitoring the natural course of the disease, particularly in patients with mild disease, but also for the assessment of therapeutic interventions. This is of particular interest in children, since ongoing bacterial infection needs to be treated vigorously to prevent neutrophilic inflammation that might lead to lung damage prior to the onset of pulmonary function impairment. Even the two patients, in whom no increased neutrophil numbers (compared to healthy controls) were apparent in the pooled BAL fluid, showed increased numbers of granulocytes in all sputum fractions. Future studies could prospectively evaluate the effect of antiinflammatory treatment on sputum cytology, similarly to asthma, for follow-up of children with CF at an early stage of the disease $[20,21]$.

In conclusion, differential cell counts obtained from three consecutive fractions of induced sputum as well as from the first and pooled fractions of bronchoalveolar lavage fluid indicated neutrophilic inflammation within the airways of children with mild cystic fibrosis. The results were consistent in suggesting that the percentage of neutrophils decreased with increasing depth of the airways. Sputum induction turned out to be safe and reproducible as regards the assessment of neutrophils. Thus, sputum induction should be the subject of future studies as a valid sensitive noninvasive tool for monitoring airway inflammation in children with cystic fibrosis and mild lung disease.

\begin{abstract}
Acknowledgements. The authors would like to thank U. Schneider (Charité, Humboldt University, Berlin, Germany) for assessment of differential cell counts in bronchoalveolar lavage fluid during the course of the BEAT study.

Members of the Bronchoalveolar Lavage for Evaluation of Anti-inflammatory Treatment study group: $F$. Ratjen (Children's Hospital, University of Essen, Essen, Germany), E. Rietschel (Children's Hospital, University of Cologne, Cologne, Germany), M. Griese (Children's Hospital, University of Munich, Munich, Germany), M. Ballmann (Medical School, University of Hannover, Hannover, Germany), G. Döring (Hygiene Institute, University of Tübingen, Tübingen, Germany), D. Reinhardt (Humboldt University, Berlin, Germany), U. Wahn (Charité, Humboldt University, Berlin, Germany), H. von der Hardt (Medical School, University of Hannover, Hannover, Germany) and K. Paul (Charité, Humboldt University, Berlin, Germany), the director of this study.
\end{abstract}

\section{References}

1. Konstan MW, Berger M. Current understanding of the inflammatory process in cystic fibrosis: onset and etiology. Pediatr Pulmonol 1997; 24: 137-142.

2. Silverman M, Pedersen S, Grigg J. Measurement of airway inflammation in young children. Am J Respir Crit Care Med 2000; 162: S1.

3. Ratjen F, Rietschel E, Griese M, et al. Fractional analysis of bronchoalveolar lavage fluid cytology in cystic fibrosis patients with normal lung function. Eur Respir J 2000; 15: 141-145.

4. Haslam PL, Baughman RP. Report of ERS Task Force: guidelines for measurement of acellular components and standardization of BAL. Eur Respir J 1999; 14: 245-248.

5. Jayaram L, Parameswaran K, Sears MR, Hargreave FE. Induced sputum cell counts: their usefulness in clinical practice. Eur Respir $J$ 2000; 16: 150-158.

6. Magnussen $\mathrm{H}$, Holz $\mathrm{O}$. Monitoring airway inflammation in asthma by induced sputum. Eur Respir J 1999; 13: 5-7.

7. Pizzichini E, Pizzichini MM, Efthimiadis A, et al. Indices of airway inflammation in induced sputum: reproducibility and validity of cell and fluid phase measurements. Am J Respir Crit Care Med 1996; 154: 308-317.

8. Keatings VM, Evans DJ, O'Connor BJ, Barnes PJ. Cellular profiles in asthmatic airways: a comparison of induced sputum, bronchial washings, and bronchoalveolar lavage. Thorax 1997; 52: 372-374.

9. Maestrelli P, Saetta M, Di Stefano A, et al. Comparison of leukocyte counts in sputum, bronchial biopsies, and bronchoalveolar lavage. Am J Respir Crit Care Med 1995; 152: 1926-1931.

10. De Boeck K, Alifier M, Vandeputte S. Sputum induction in young cystic fibrosis patients. Eur Respir J 2000; 16: 9194.

11. Sagel SD, Kapsner R, Osberg I, Sontag MK, Accurso FJ. Airway inflammation in children with cystic fibrosis and healthy children assessed by sputum induction. Am J Respir Crit Care Med 2001; 164: 1425-1431. 
12. Ratjen F, Bredendiek M, Brendel M, Meltzer J, Costabel U. Differential cytology of bronchoalveolar lavage fluid in normal children. Eur Respir J 1994; 7: 1865-1870.

13. Gershman NH, Liu H, Wong HH, Liu JT, Fahy JV. Fractional analysis of sequential induced sputum samples during sputum induction: evidence that different lung compartments are sampled at different time points J Allergy Clin Immunol 1999; 104: 322-328.

14. Holz O, Jörres RA, Koschyk S, Speckin P, Welker L, Magnussen $\mathrm{H}$. Changes in sputum composition during sputum induction in healthy and asthmatic subjects. Clin Exp Allergy 1998; 28: 284-292.

15. Brunner E, Domhof S, Langer F. Nonparametric Analysis of Longitudinal Data in Factorial Designs. New York, Wiley, 2001.

16. Bartko JJ. Measurements and reliability: statistical thinking considerations. Schizophr Bull 1991; 17: 483-489.
17. Wong HH, Faby W. Safety of one method of sputum induction in asthmatic subjects. Am J Respir Crit Care Med 1997; 155: 299-303.

18. Moodley YP, Krishnan V, Lalloo UG. Neutrophils in induced sputum arise from central airways. Eur Respir $J$ 2000; 15: 36-40.

19. Alexis NE, Hu SC, Zeman K, Alter T, Bennett WD. Induced sputum derives from the central airways. Confirmation using a radiolabeled bolus delivery technique. Am J Respir Crit Care Med 2001; 164: 1964-1970.

20. Henig NR, Tonelli MR, Pier MV, Burns JL, Aitken ML. Sputum induction as a research tool for sampling the airways of subjects with cystic fibrosis. Thorax 2001; 56: 306-311.

21. Oh JW, Lee HB, Kim CR, et al. Analysis of induced sputum to examine the effect of inhaled corticosteroid on airway inflammation in children with asthma. Ann Allergy Asthma Immunol 1999; 82: 491-496. 